\title{
PAST and FUTURE
}

with GIAI?! 

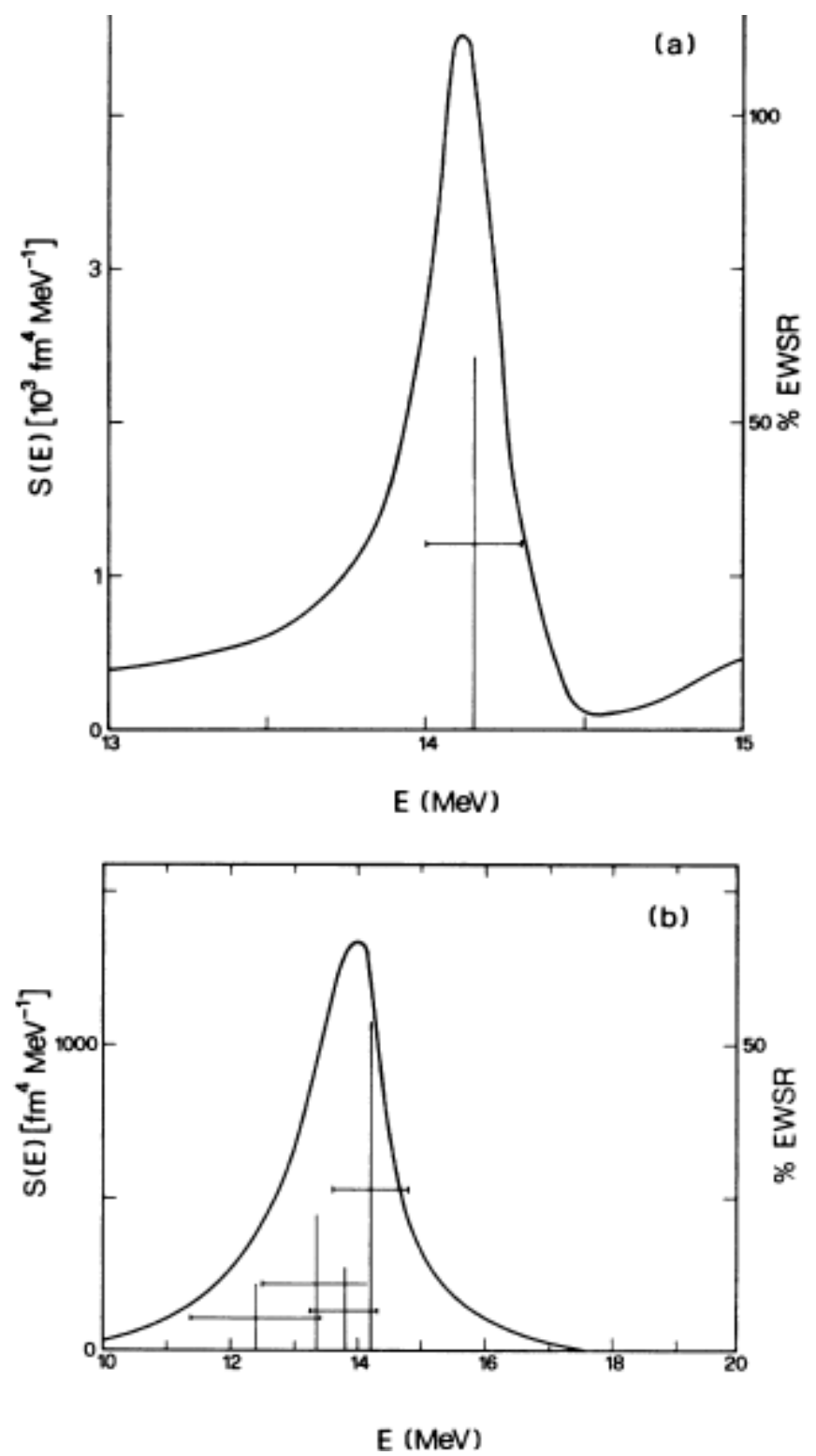


\title{
Study of the Breathing Mode of ${ }^{208} \mathrm{~Pb}$ through Neutron Decay
}

\author{
A. Bracco \\ Dipartimento di Fisica, Università di Milano, Milano, Italy, and Istituto Nazionale di Fisica Nucleare, \\ Sezione di Milano, I-20133 Milano, Italy \\ J. R. Beene \\ Physics Division, Oak Ridge National Laboratory, Oak Ridge, Tennesee 37830 \\ N. Van Giai \\ Division de Physique Théorique, Institut de Physique Nucleaire, F-91406 Orsay Cedex, France

$$
\text { P. F. Bortignon }
$$ \\ Istituto di Ingegneria Nucleare, Centro Studi Nucleari Enrico Fermi, Politecnico di Milano, \\ I-20133 Milano, Italy, and Laboratori Nazionali di Legnaro, Istituto Nazionale di Fisica Nucleare, \\ I-35020 Legnaro, Italy$$
\text { F. Zardi }
$$ \\ Istituto Nazionale di Fisica Nucleare, Sezione di Padova, I-35131 Padova, Italy
}

and

\section{R. A. Broglia}

Dipartimento di Fisica, Università di Milano, Milano, Italy, and Istituto Nazionale di Fisica, Sezione di Milano, I-20133 Milano, Italy, and The Niels Bohr Institute, University of Copenhagen, Copenhagen, Denmark (Received 31 March 1988) 
$\mathcal{H}(\omega) \equiv Q_{1} H Q_{1}+W^{\dagger}(\omega)+W^{\downarrow}(\omega)=Q_{1} H Q_{1}+Q_{1} H P \frac{1}{\omega-P H P+i \epsilon} P H Q_{1}+Q_{1} H Q_{2} \frac{1}{\omega-Q_{2} H Q_{2}+i \epsilon} Q_{2} H Q_{1}$,

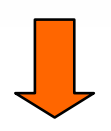

RPA

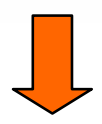

continuum coupling

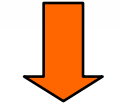

1p-1h-1 phonon

coupling

This effective Hamiltonian can be diagonalized and from its eigenvalues and eigenvectors one can extract the response function to a given operator $\mathrm{O}$.

$$
R(\omega)=\left\langle 0\left|O^{\dagger} \frac{1}{\omega-\mathcal{H}(\omega)+i \epsilon} O\right| 0\right\rangle
$$

$$
S(\omega)=-\frac{1}{\pi} \operatorname{Im} R(\omega) .
$$

$$
S(\omega)=-\frac{1}{\pi} \operatorname{Im} \sum_{\nu}\langle 0|O| \nu\rangle^{2} \frac{1}{\omega-\Omega_{\nu}+i \frac{\Gamma_{\nu}}{2}}
$$

It is possible to extract at the same time to calculate the branching ratios associated with the decay of the GR to the $\mathrm{A}-1$ nucleus in the channel c (hole state).

$$
B_{c}(\omega) \equiv \frac{\sigma_{c}(\omega)}{\sigma_{\text {exc }}(\omega)}=\frac{\sum_{\nu, \nu^{\prime}} S_{\nu^{\prime} \nu} \gamma_{\nu^{\prime}, \nu}\left(\omega-\Omega_{\nu}-i \frac{\Gamma_{\nu}}{2}\right)^{-1}\left(\omega-\Omega_{\nu^{\prime}}+i \frac{\Gamma_{\nu^{\prime}}}{2}\right)^{-1}}{-2 \operatorname{Im} \sum_{\nu, \nu^{\prime}} S_{\nu^{\prime} \nu}\left(F^{*} F^{T}\right)_{\nu \nu^{\prime}}\left(\omega-\Omega_{\nu^{\prime}}-i \frac{\Gamma_{\nu^{\prime}}}{2}\right)^{-1}}
$$




\section{The IAS: a stringent test}

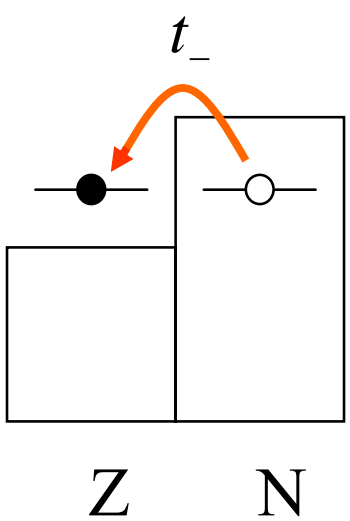

\begin{tabular}{|c|c|c|c|c|c|c|c|c|c|}
\hline & \multicolumn{2}{|c|}{ (a) Discrete TDA } & \multicolumn{3}{|c|}{ (b) $\mathrm{RPA}+W^{\dagger}$} & \multicolumn{4}{|c|}{ (c) $\mathrm{RPA}+{ }_{W}^{\dagger}+{ }_{W}$} \\
\hline & $E$ IAR & $\%$ of $m 0$ & $E$ LAR & $\Gamma^{\dagger}$ & $\%$ of $m 0$ & $E$ LAR & $\Gamma_{\text {tot }}$ & $\Gamma^{+}$ & $\%$ of $m 0$ \\
\hline I & 0.268 & 99.9 & - & - & - & 0.267 & 24 & 24 & 99.7 \\
\hline \multirow[t]{2}{*}{ II } & 18.50 & 85 & 18.50 & 124 & 97 & 18.36 & 194 & 70 & 97 \\
\hline & 18.28 & 16 & & & & & & & \\
\hline \multirow[t]{2}{*}{ III } & 18.64 & 80 & 18.65 & 128 & 96 & 18.54 & 228 & 100 & 96 \\
\hline & 18.39 & 11 & & & & & & & \\
\hline
\end{tabular}

The measured total width $\left(\Gamma_{\exp }=230 \mathrm{keV}\right)$ is well reproduced. The accuracy of the symmetry restoration (if $\mathrm{V}_{\text {coul }}=0$ ) can be established.

\begin{tabular}{|c|c|c|c|c|c|}
\hline \multirow{3}{*}{$\begin{array}{l}\text { Decay } \\
\text { channel }\end{array}$} & \multicolumn{4}{|c|}{ Theory } & \multirow{3}{*}{ Experiment } \\
\hline & \multirow[t]{2}{*}{ Only $W^{\dagger}$} & \multicolumn{3}{|c|}{$W^{\dagger}+W^{\downarrow}$} & \\
\hline & & (a) & (b) & (c) & \\
\hline$p_{1 / 2}$ & 0.472 & 0.346 & 0.253 & 0.237 & $0.22 \pm 0.02$ \\
\hline$p_{3 / 2}$ & 0.396 & 0.287 & 0.238 & 0.196 & $0.34 \pm 0.04$ \\
\hline$i_{13 / 2}$ & 0.015 & 0.011 & 0.008 & 0.010 & - \\
\hline$f_{5 / 2}$ & 0.117 & 0.086 & 0.065 & 0.061 & included in $p_{3 / 2}$ \\
\hline$f_{7 / 2}$ & $<10^{-3}$ & $<10^{-3}$ & $<10^{-3}$ & $<10^{-3}$ & $0.015 \pm 0.007$ \\
\hline$h_{\vartheta / 2}$ & $<10^{-3}$ & $<10^{-3}$ & $<10^{-3}$ & $<10^{-3}$ & - \\
\hline$\sum_{c} B_{c}$ & 1.0 & 0.730 & 0.564 & 0.504 & $0.575 \pm 0.07$ \\
\hline
\end{tabular}




\section{DAMPING OF COLLECTIVE MODES}

Hierarchy of couplings for damping of giant resonances : from mean field states to Compound Nucleus

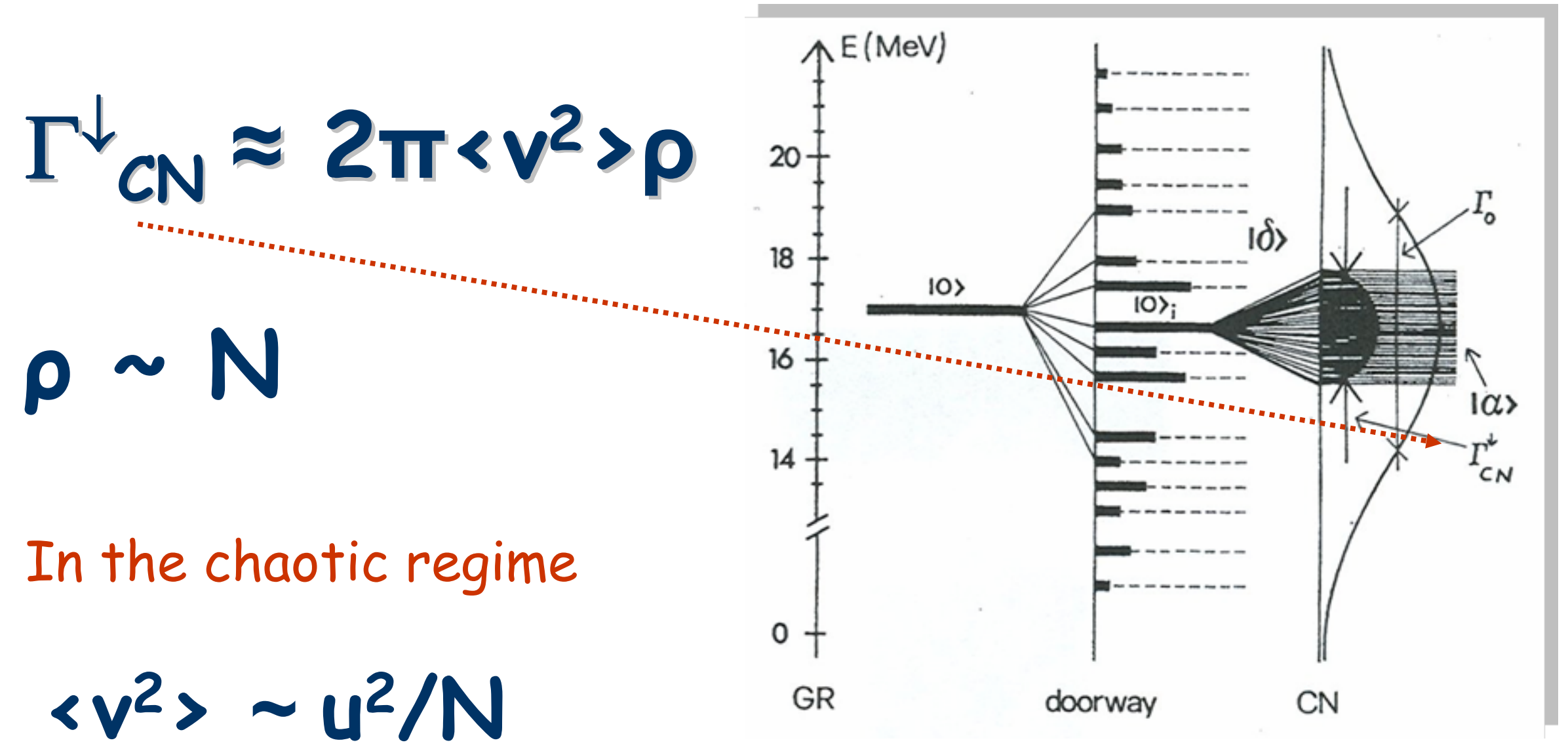


PHYSICAL REVIEW C 77, 024302 (2008)

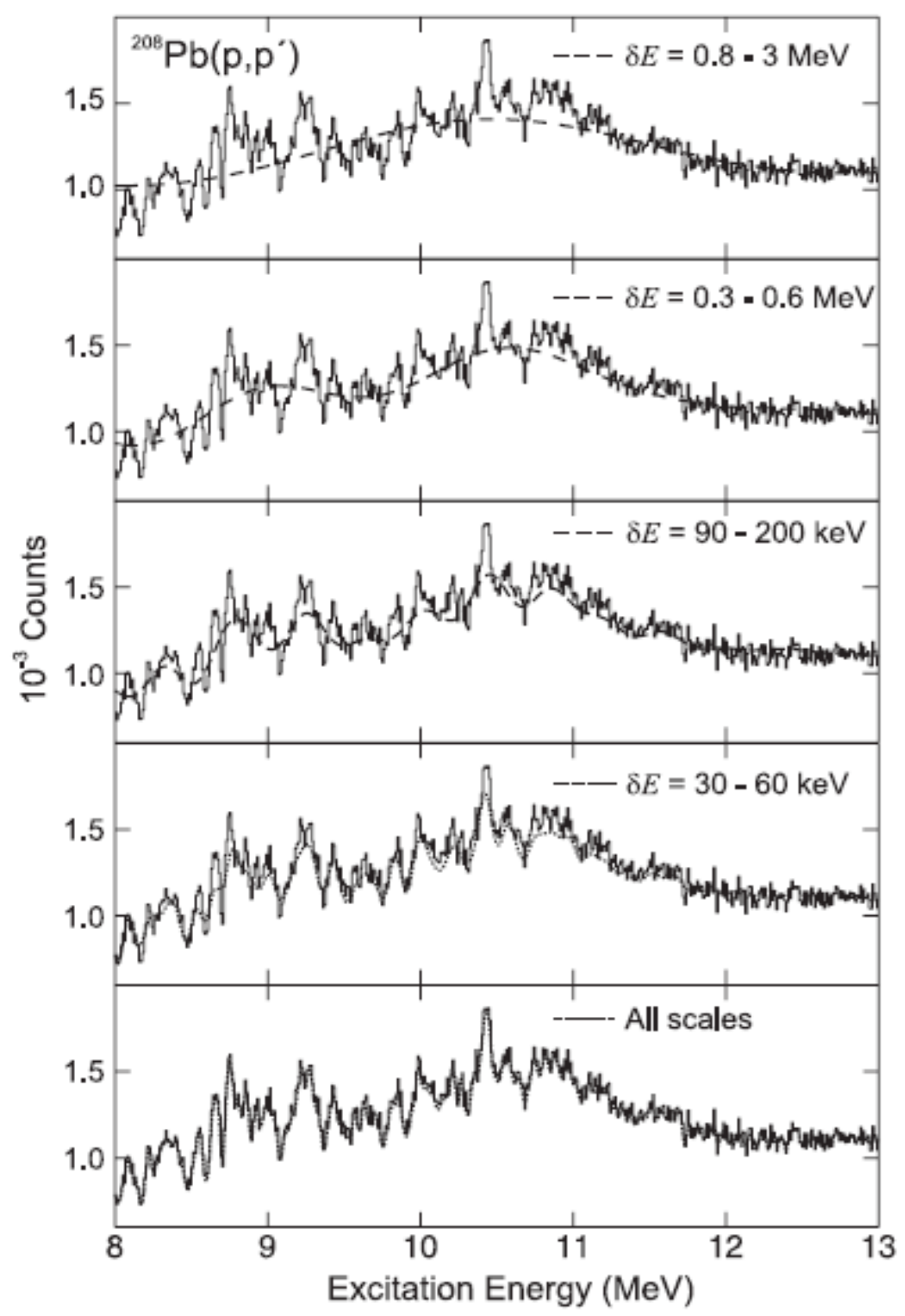




\section{Sn low-lying GDR strength}

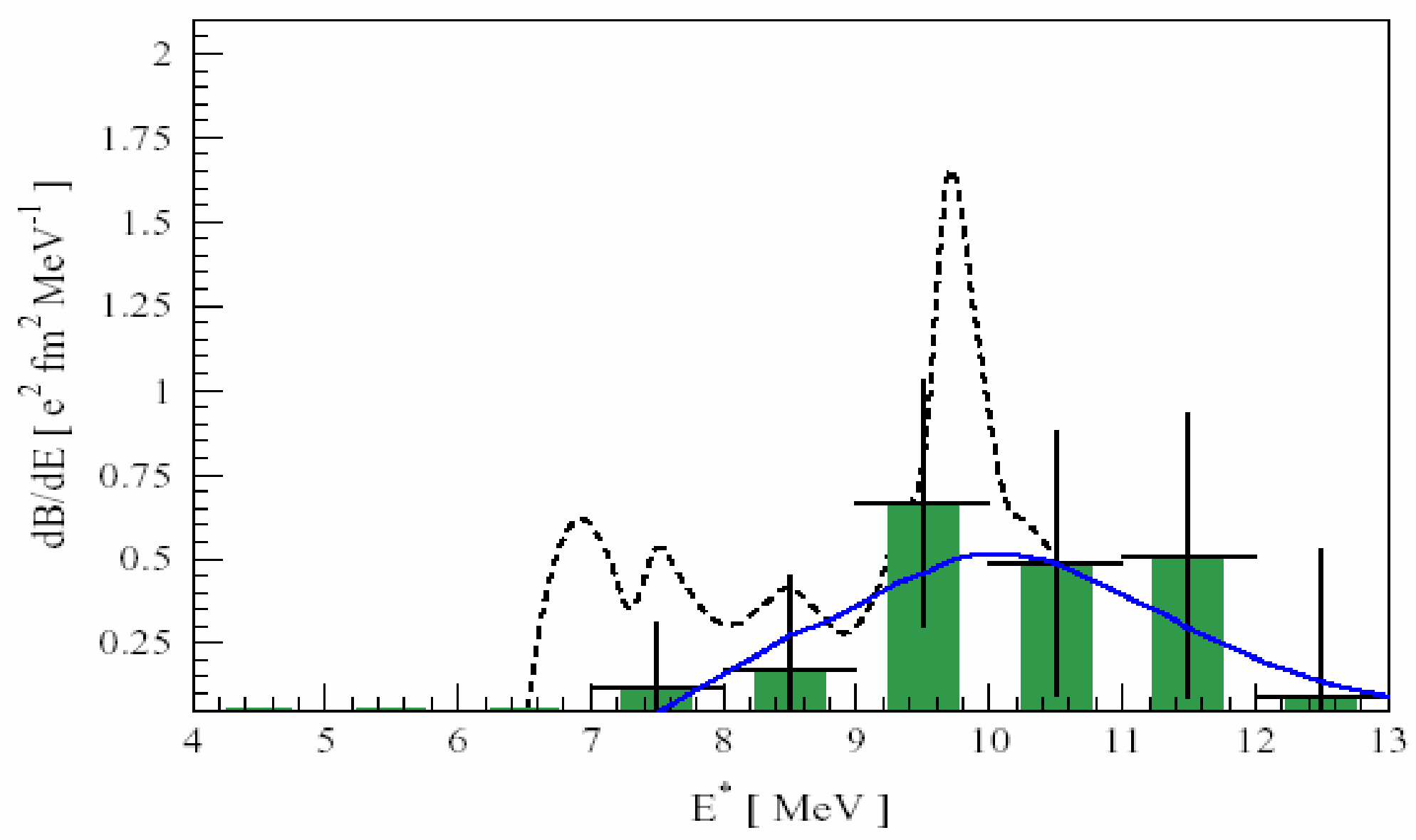


Dipole strength shifts at low energy.

Collective or non-collective nature of the transitions?
In neutron rich coulomb excited ${ }^{68} \mathrm{Ni}$ a structure centered at $\sim 10.5 \mathrm{MeV}$ has been measured in the $\gamma$-ray spectra
Stable nuclei $\Rightarrow$ photoabsorption

Exotic nuclei

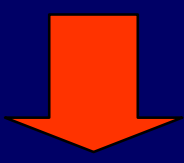

Virtual photon breakup

LAND experiment

Aldrich PRL95(2005)132501

Virtual photon scattering

RISING experiment
Measured $\gamma$-ray spectra

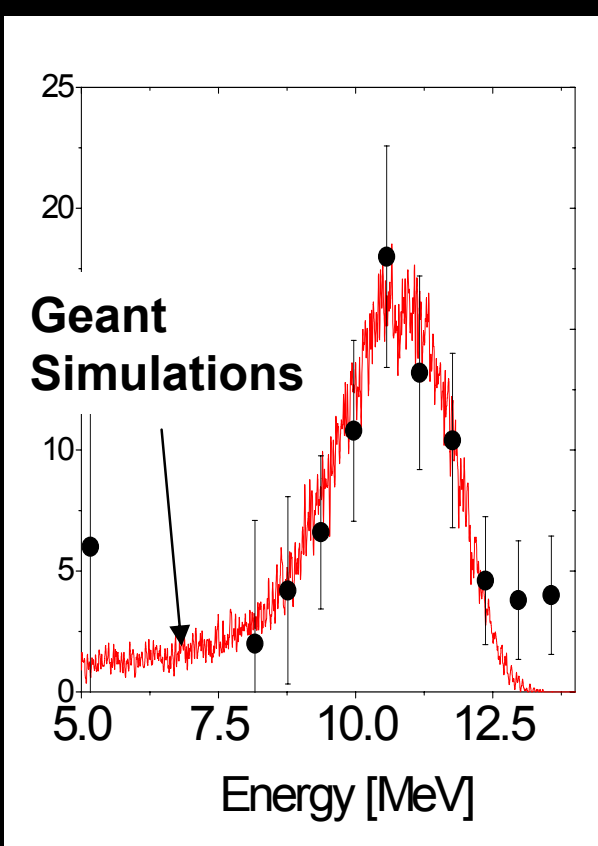

Theory

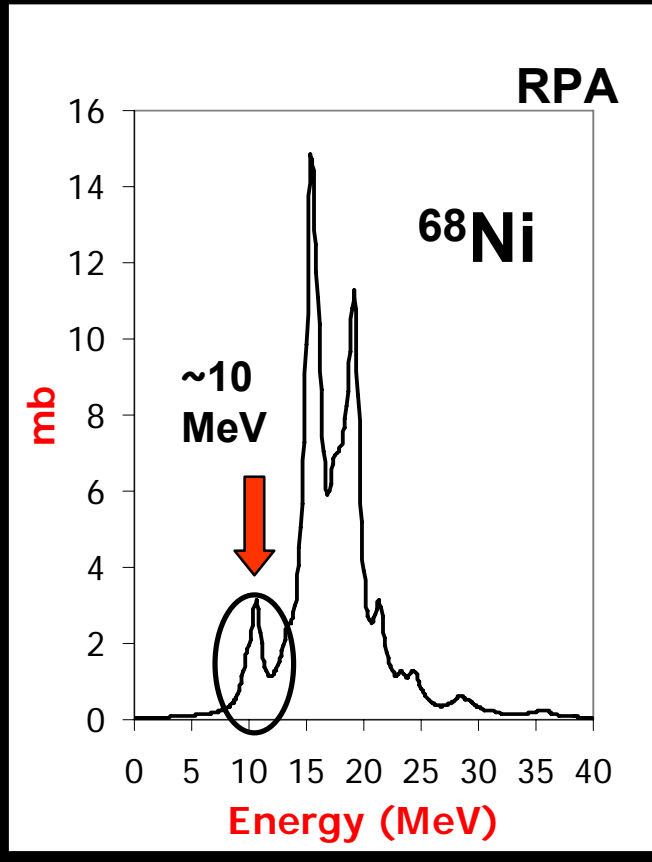


Going beyond mean field: medium polarization effects

Self-energy

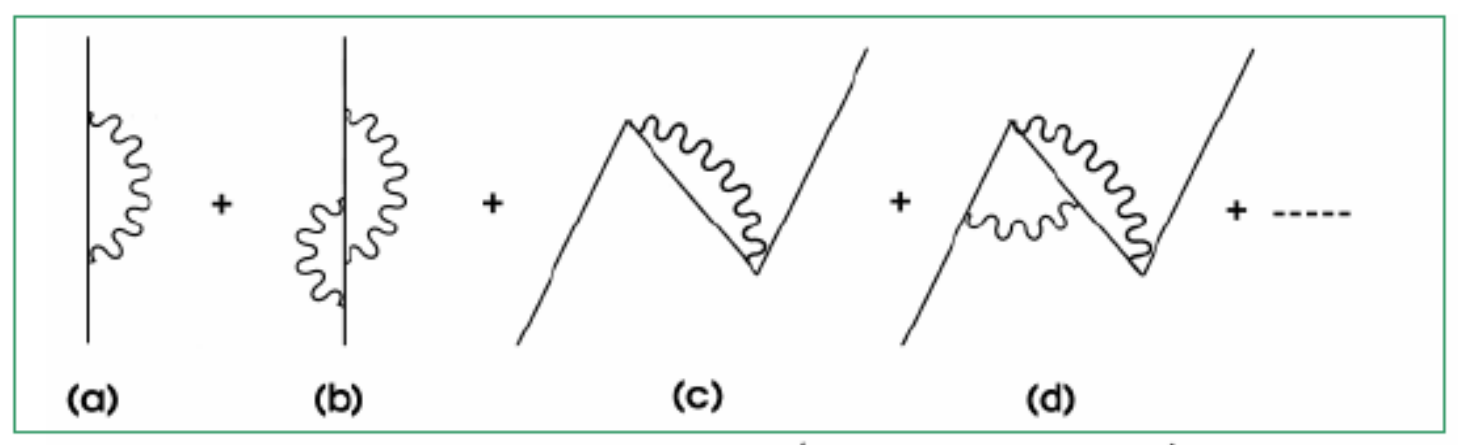

Induced interaction (screening)

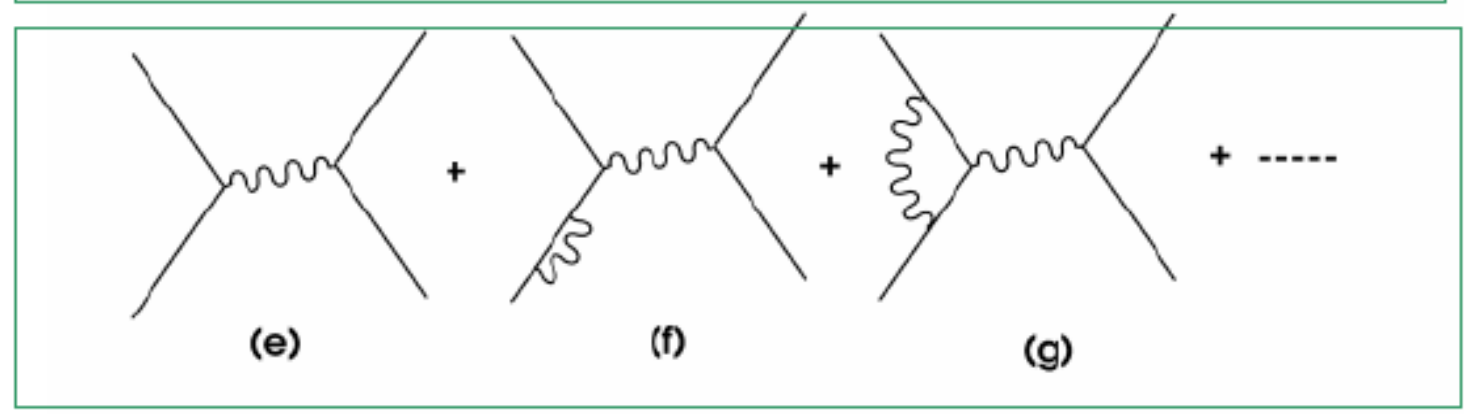


The empirical evidence on single-particle states can be summarized as follows: For $\left|\epsilon_{\nu}-\epsilon_{F}\right|>10-15 \mathrm{MeV}$,

$$
m^{*} / m \approx 0.7 \quad ; \quad \Sigma_{\nu}(\omega)=\Delta E_{\nu}-\frac{i}{2} \Gamma_{\nu}(\omega)
$$

and for $\left|\epsilon_{\nu}-\epsilon_{F}\right|<10-15 \mathrm{MeV}$,

$$
m^{*} / m \approx 1 \quad ; \quad \Gamma \approx 0 \quad Z_{\omega} \approx 0.6-0.7
$$

In the equations above, $m$ is the bare nucleon mass, $m^{*}$ the effective mass and $\Sigma_{\nu}(\omega)$ is the self-energy operator of the single-particle state with quantum numbers $\nu$. The centroid of the corresponding strength function being $\epsilon_{\nu}+$ $\Delta E_{\nu}$, the width $\Gamma_{\nu} \approx 0.5\left|\epsilon_{\nu}-\epsilon_{F}\right| ; Z_{\omega}$ denotes the discontinuity at the Fermi energy, which is closely related to the spectroscopic factors of valence orbitals. Below the Fermi energy they are not completely filled, above not completely empty.

One can approach the calculation of the mean field the way one prefers. However, once the corresponding Hamiltonian has been diagonalized, it should reproduce the empirical facts (1) and (2) recalled above. 
C. Maibaux er af, Dynamies of the shell modes

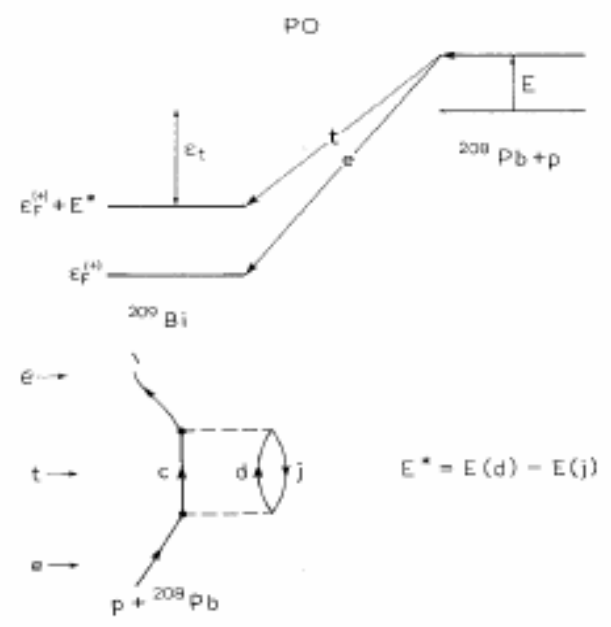

C. Mahaux er al., Dynamics of the shell model

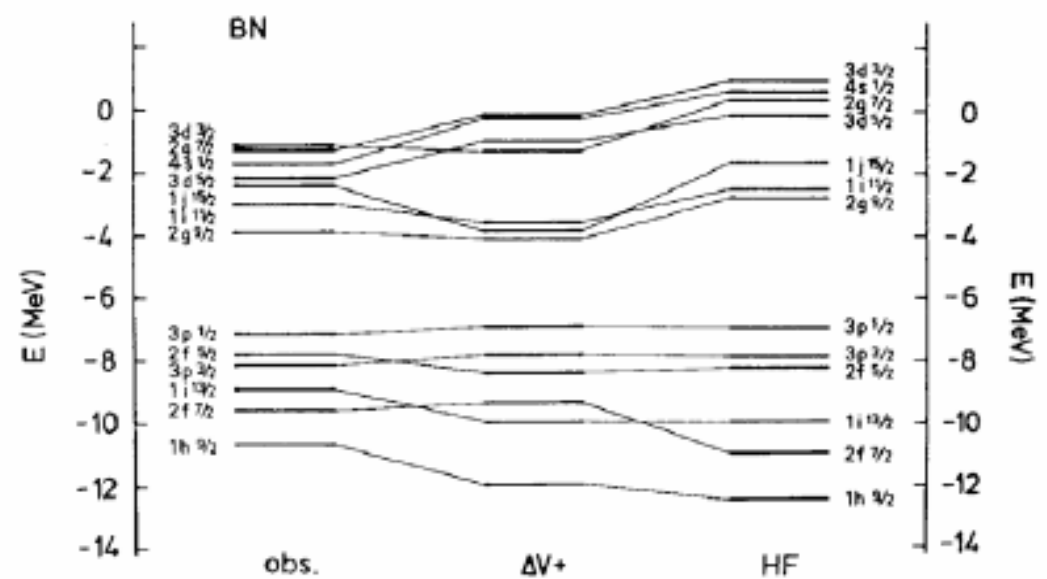

Coupling of vibrations to single-particle motion

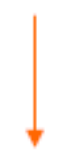

\section{Effective mass $m_{\omega}$}

Increased density at the Fermi energy 


\section{On Self-Energy, Effective Masses, Level Density}

$$
\begin{gathered}
\Delta E_{\beta}(\omega)=\sum_{\alpha} \frac{V_{p v}^{2}(\alpha, \beta ; L)}{\omega-\left(\epsilon_{\alpha}+\hbar \omega_{L}\right)} \\
\frac{d \epsilon}{d k}=\frac{\hbar^{2} k}{m^{*}} \\
\frac{m^{*}}{m}=\frac{m_{k}}{m} \frac{m_{\omega}}{m} \\
m_{k}=m\left(1+\frac{m}{\hbar^{2}} \frac{\partial U_{H F}}{\partial k}\right)^{-1} \\
m_{\omega}=m\left(1-\frac{\partial \Delta E}{\partial \omega}\right) \\
\left(\frac{\partial \Delta E}{\partial \omega}\right)_{\omega=0} \approx-N(0) \int_{0}^{\infty} \frac{V_{p v}^{2} d \epsilon}{\left(\epsilon+\hbar \omega_{L}\right)^{2}}=-N(0) \frac{V_{p v}^{2}}{\hbar \omega_{L}}
\end{gathered}
$$


We obtain also the quasi-particle strength (spectroscopic factor)

$$
Z_{\omega}=\left(M_{\omega} / m\right)^{-1}
$$

In the Fermi gas model, the level density reads

$$
\rho\left(A, E^{*}\right) \propto \exp \left(2 \sqrt{a E^{*}}\right)
$$

being

$$
a \propto A / \epsilon_{F} \propto m^{*}
$$




\section{Going beyond the quasi-particle approximation}

\section{J. Terasaki et al., Nucl.Phys. A697(2002)126}

by extending the Dyson equation...

$$
G_{\mu}^{-1}=\left(G_{\mu}^{0}\right)^{-1}-\sum_{\mu}(\omega)
$$

to the case of superfluid nuclei (Nambu-Gor'kov), it is possible to consider both

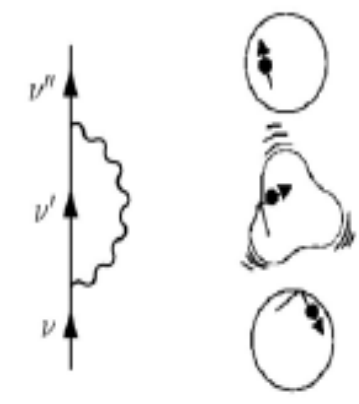

and

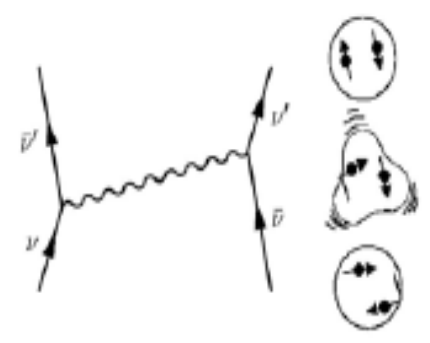



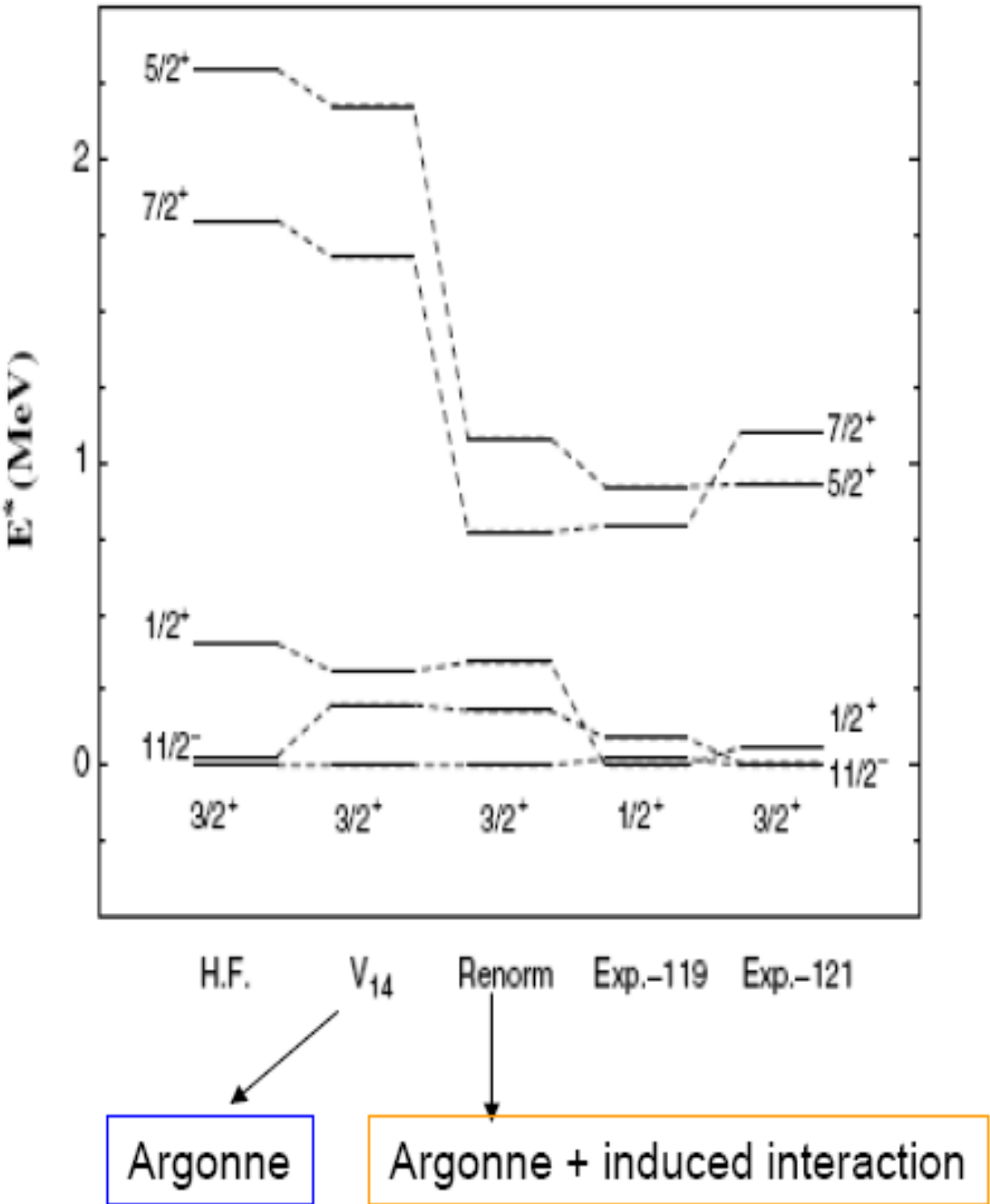

F. Barranco et al., EPJA21(2004)57 


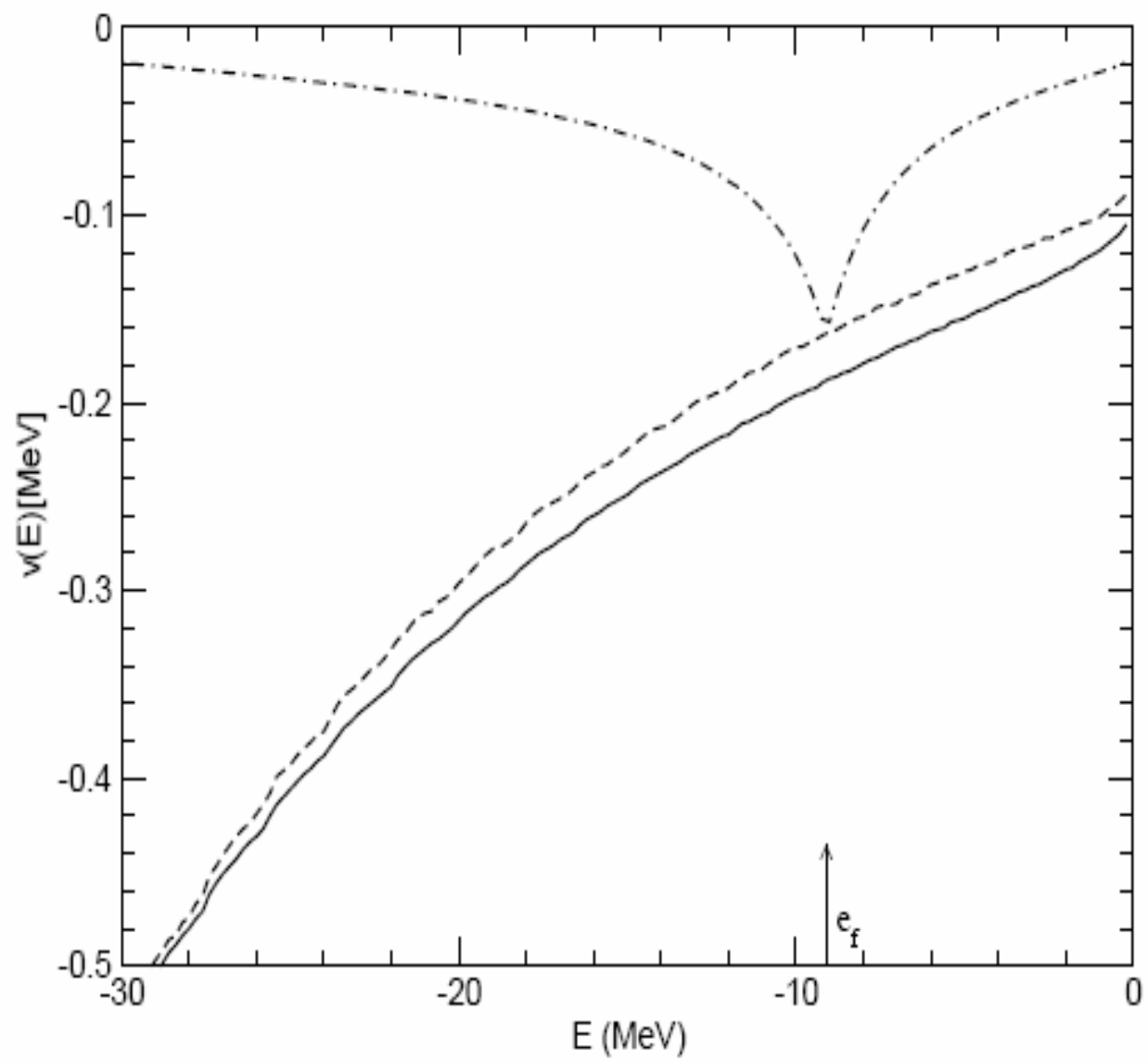

Figure 5: The nucleus ${ }^{120} \mathrm{Sn}$. The semiclassical matrix elements of the induced interaction, calculated according to Eq. (9)(dash-dotted curve), are compared with the matrix elements of the Gogny force (solid curve, cf. Fig. 3) and with those of the $v_{l o w-k}$ interaction (dashed curve). Calculations are performed with $m_{k}=m$ and with the same Woods-Saxon potential used in Figs. 1 and 3.

\section{F. Barranco....P.Schuck et al., PRC 72}



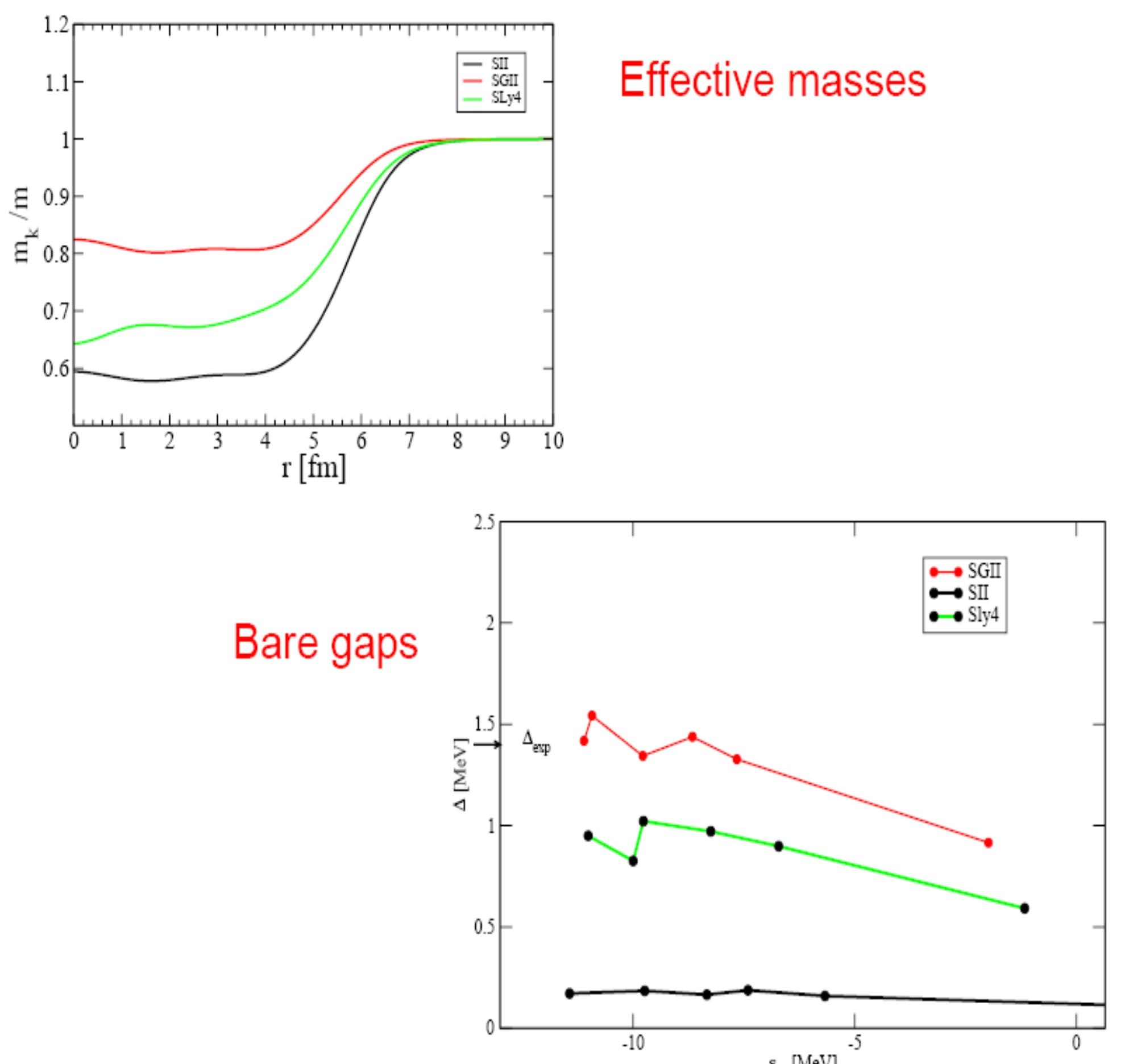


\section{Renormalization of pairing gaps}

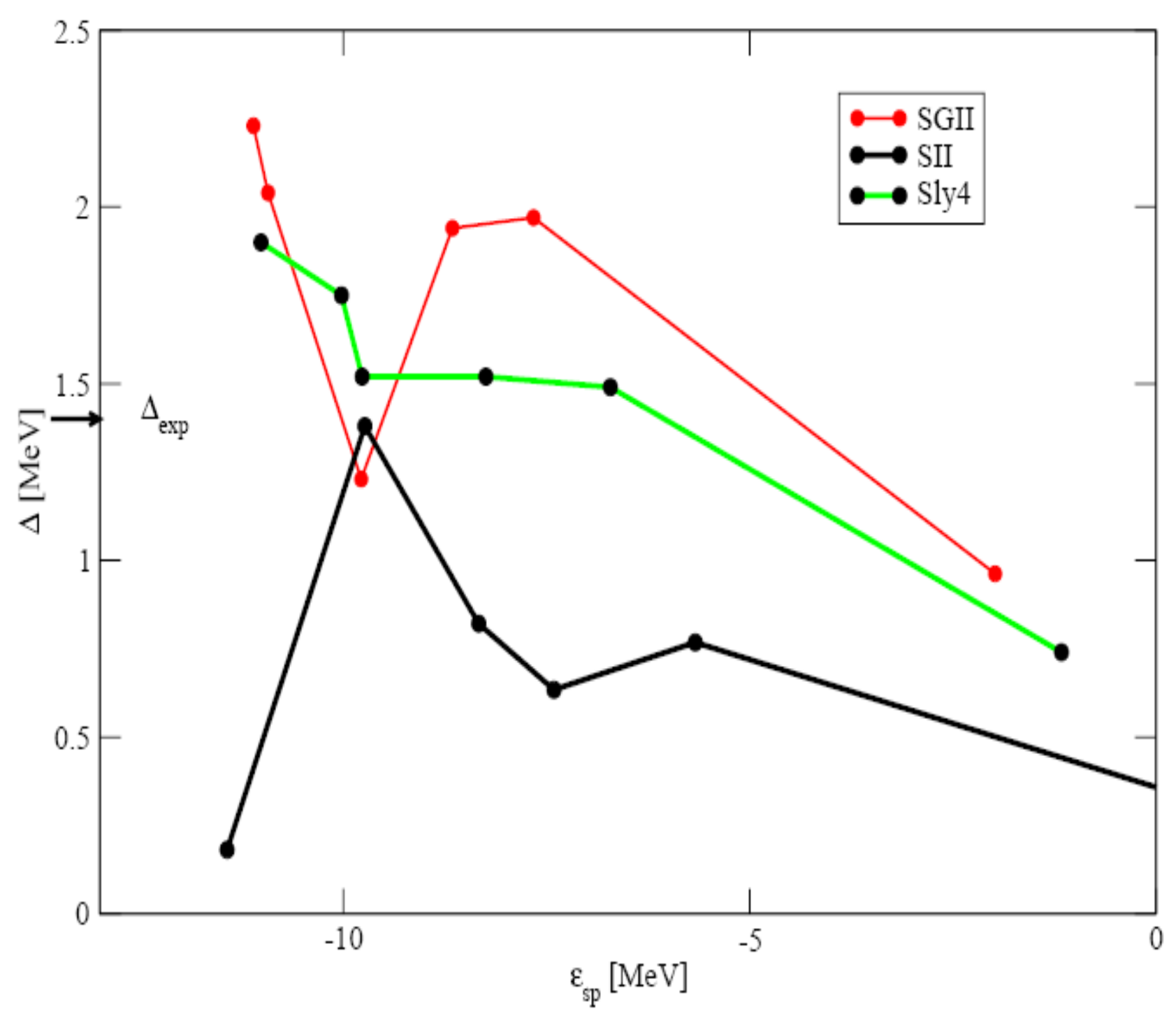




\section{$d_{3 / 2}$}

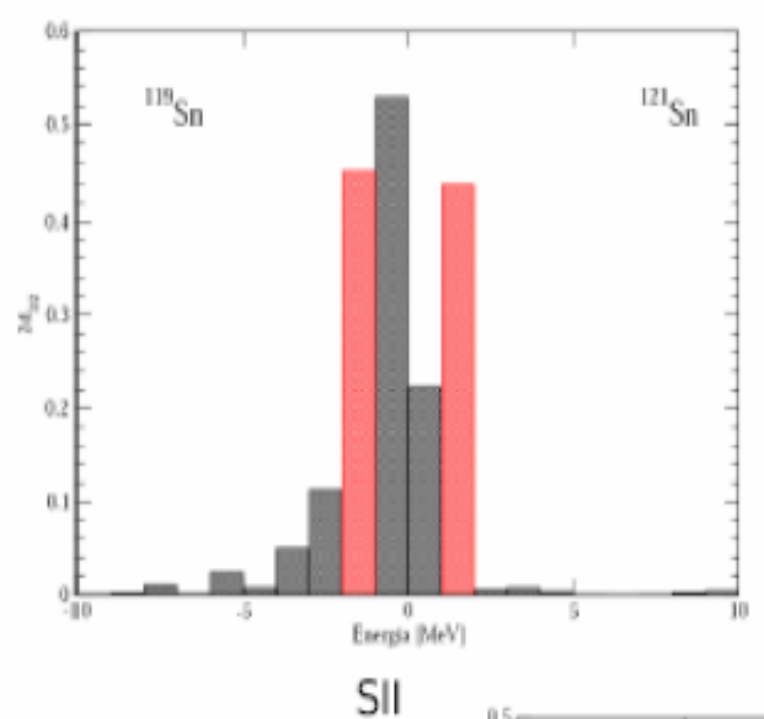

Exp.

Th.
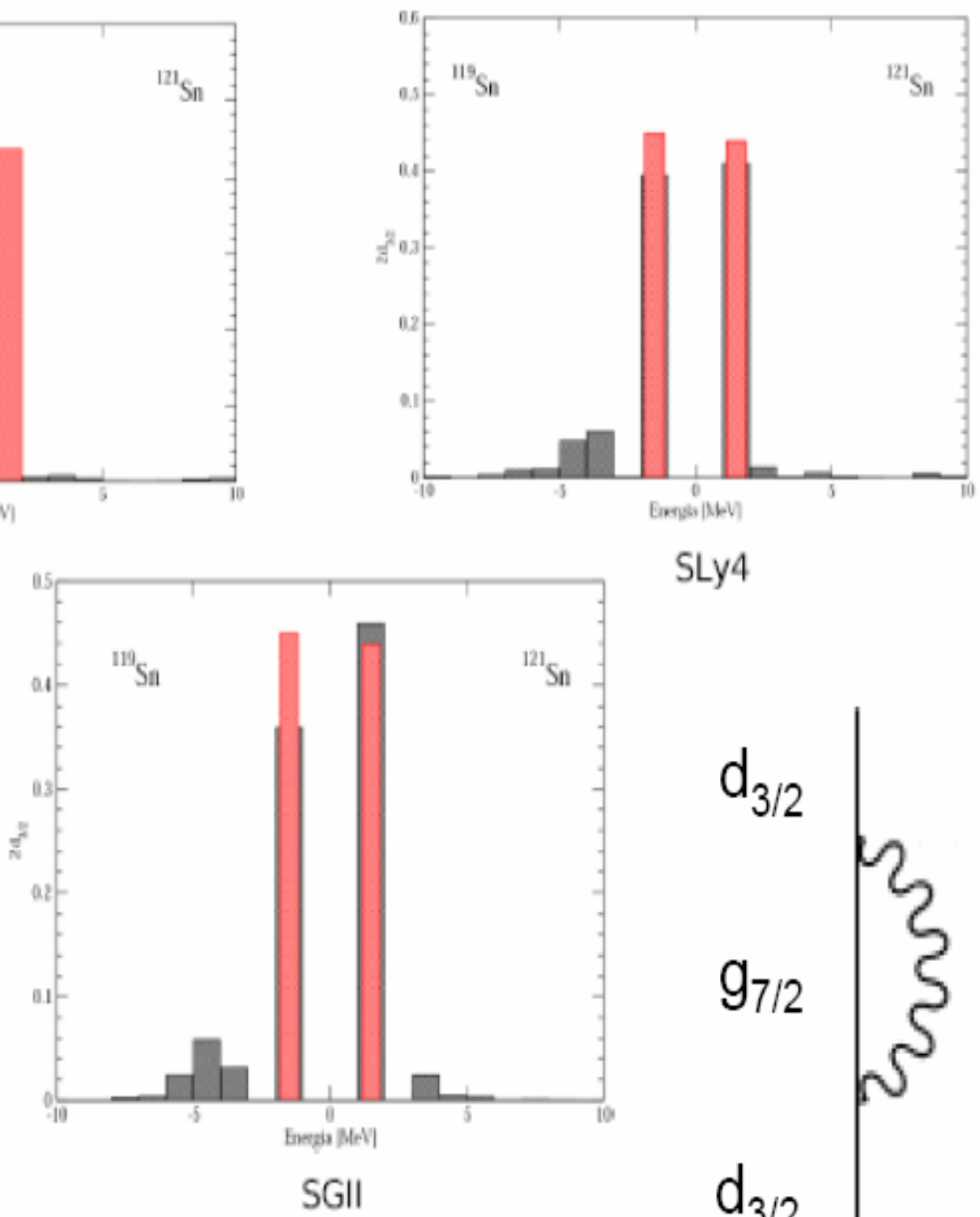

SLy4

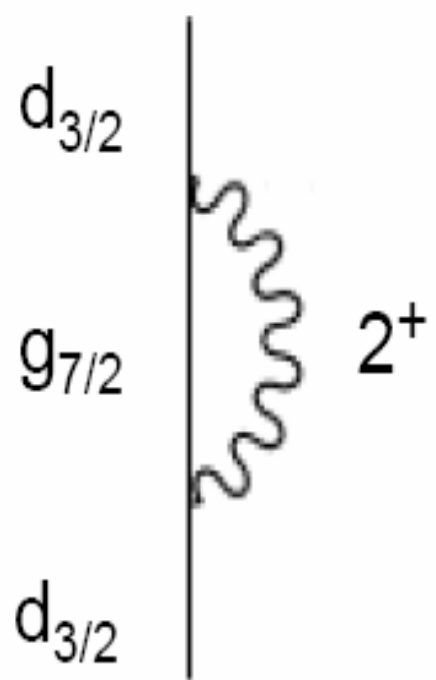




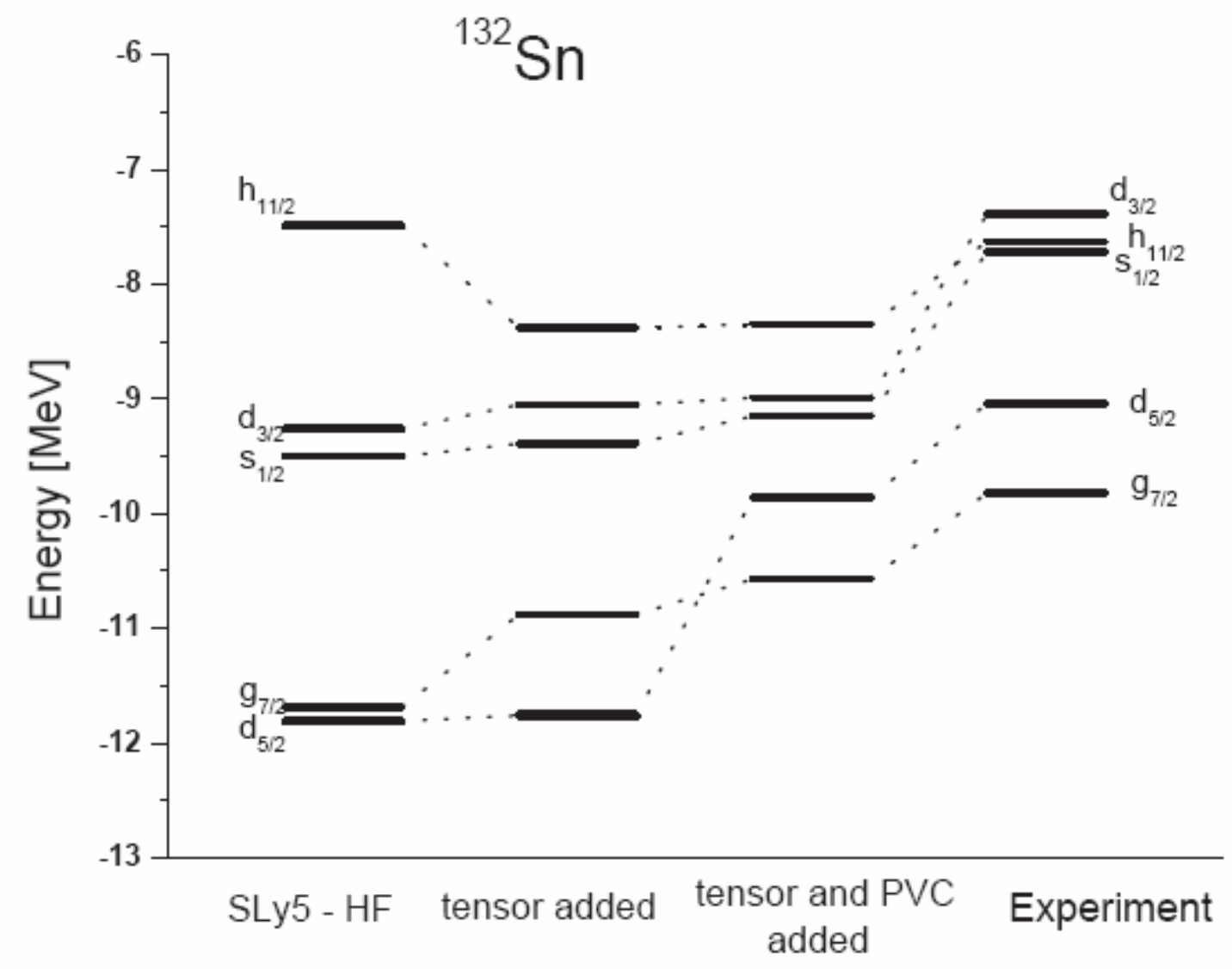




\section{Paul Bonche et al.}

- Let us emphasize that this study was done at the mean-field (HF) level. However, NO ingredient in our protocol prevents further studies beyond the mean field approximation. If needed be, further correlations can be explored and it is quite legitimate to use these interactions for RPA or configurations mixing (GCM) calculations. This would NOT has been the case if we had included in our protocol detailed information such as s.p. energies of some selected nuclei. 


\title{
Dependence of single-particle energies on coupling constants of the nuclear energy density functional
}

\author{
M. Kortelainen, ${ }^{1}$ J. Dobaczewski,${ }^{1,2}$ K. Mizuyama, ${ }^{1}$ and J. Toivanen ${ }^{1}$ \\ ${ }^{1}$ Department of Physics, P.O. Box 35 (YFL), FI-40014 University of Jyväskylä, Finland \\ ${ }^{2}$ Institute of Theoretical Physics, University of Warsaw, ul. Hoża 69, 00-681 Warsaw, Poland. \\ (Dated: March 18, 2008) \\ We show that single-particle energies in doubly magic nuclei depend almost linearly on the cou- \\ pling constants of the nuclear energy density functional. Therefore, they can be very well char- \\ acterized by the linear regression coefficients, which we calculate for the coupling constants of the \\ standard Skyrme functional. We then use these regression coefficients to refit the coupling constants \\ to experimental values of single-particle energies. We show that the obtained rms deviations from \\ experimental data are still quite large, of the order of $1.1 \mathrm{MeV}$. This suggests that the current stan- \\ dard form of the Skyrme functional cannot ensure spectroscopic-quality description of single-particle \\ energies, and that extensions of this form are very much required.
}




\title{
Role of the surface in the electronic effective mass of metal microclusters
}

\author{
M. Bernath, ${ }^{1,2}$ M. S. Hansen, ${ }^{3}$ P. F. Bortignon, ${ }^{1,2}$ and R. A. Broglia ${ }^{1,2,3}$ \\ 'Dipartimento di Fisica, Università di Milano, Via Celoria 16, 20133, Milan, Italy \\ ${ }^{2}$ Istituto Nazionale di Fisica Nucleare, Sezione di Milano, Via Celoria 16, 20133, Milan, Italy \\ ${ }^{3}$ The Niels Bohr Institute, University of Copenhagen, DK-2100 Copenhagen, Denmark \\ (Received 2 June 1992; revised manuscript received 19 July 1993)
}

The renormalization of the motion of the valence electrons in metal clusters arising from the coupling to the fluctuations of the cluster surface is calculated. Sizable effects are found, which lead to renormalization coefficients which deviate $30-40 \%$ from unity. 


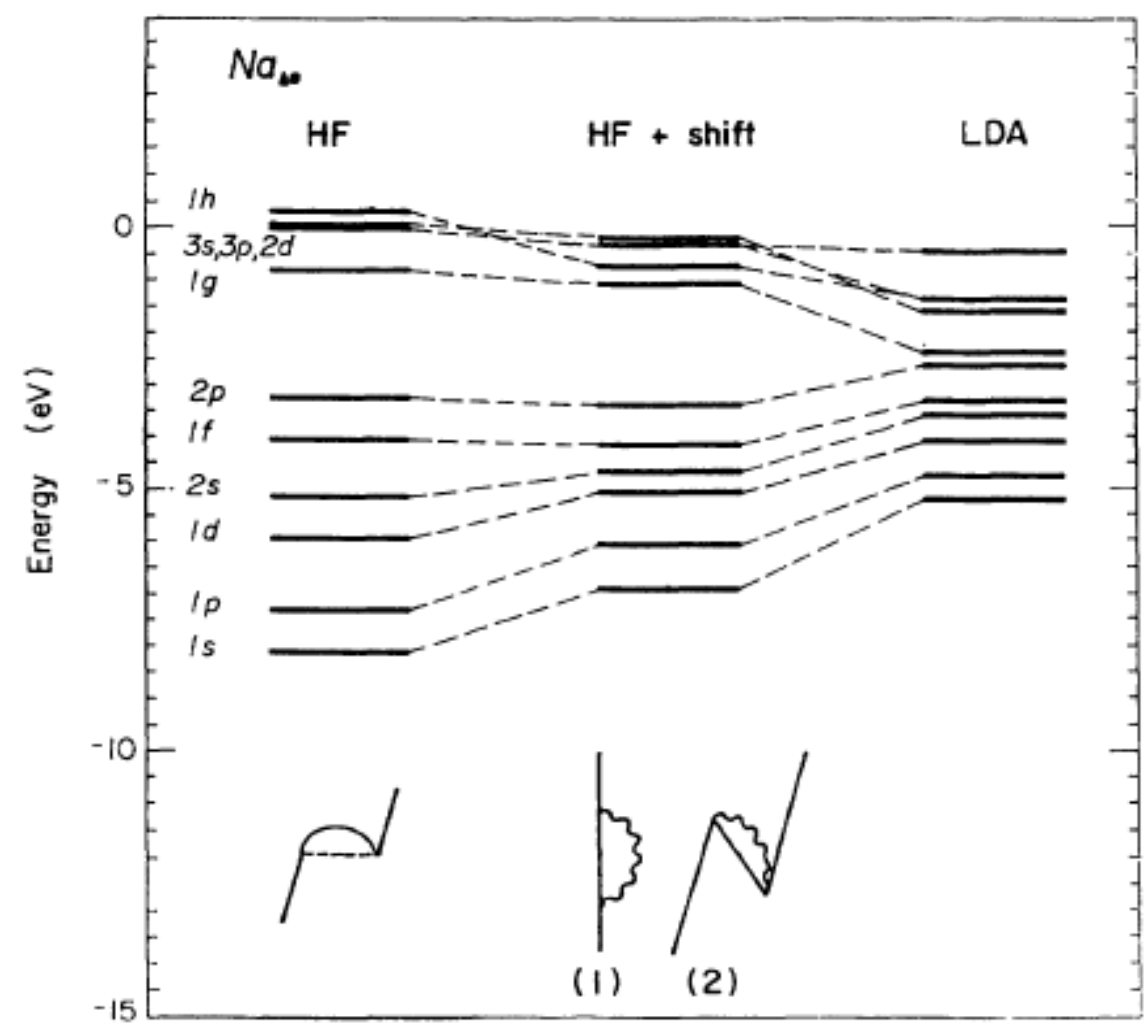

FIG. 1. First and last columns display the energy levels of $\mathrm{Na}_{40}$ within the Hartree-Fock and LDA, respectively. The middle column shows the Hartree-Fock levels corrected by the selfenergy contributions calculated as indicated in the text. In the lowest part of the figure a schematic graphical representation of the exchange (first column) and screening terms (middle column) discussed in the text is shown. 
INSERT CONSTRAINTS FROM COLLECTIVE EXCITED STATES in the fit of the functional. Concrete proposal : $K_{\infty}, S\left(\rho=0.1 \mathrm{fm}^{-3}\right), g 0^{\prime}$.

- Giant Monopole Resonance : $\mathrm{E}_{\mathrm{GMR}}$ constrains $\mathrm{K}_{\infty}=240 \pm 20 \mathrm{MeV}$. (a) Allow in the fit this relatively broad range (one can allow $1.5 \sigma$, that is, $210<\mathrm{K}_{\infty}<270 \mathrm{MeV}$ ). (b) A smaller range is possible if we have an a priori choice for the density dependence. This constraints comes from a comparative study of Skyrme, Gogny, RMF.

- Giant Dipole Resonance : $E_{\text {IVGDR }}$ constraints $S_{0.1} \equiv S\left(\rho=0.1 \mathrm{fm}^{-3}\right)$. The constraint, coming from a study with Skyrme, is $22.3<\mathrm{S}_{0.1}<25.8$ $\mathrm{MeV}$.

- Giant Quadrupole Resonance : it mainly involves m*.

- Low-lying collective states : too much dependent on s.p. spectrum.

- Giant Gamow-Teller Resonance : imposing that it exhausts about $60 \%$ of the $3(\mathrm{~N}-\mathrm{Z})$ sum rule constraints the spin-isospin part of the functional. Here the precise constraint depends on the functional.

Gianluca Colò, Univ. of Milano, Italy 